VOL. 69 (2004) [113-123]

\title{
A CONSTRUCTION OF SIMPLE LIE SUBALGEBRAS OF CERTAIN TYPES FROM TRIPLE SYSTEMS
}

\author{
Noriaki Kamiya and Susumu OkUbo
}

\begin{abstract}
We have constructed all simple Lie superalgebras from some triple systems. In particular, we shall discuss simple Lie superalgebras of $P(n), Q(n)$ and Cartan types $W(n), \widetilde{S}(n), H(n)$ in this article.
\end{abstract}

\section{i. TRIPle Systems and LiE SUPERALGebras}

It is known $([1,8])$ that all simple Lie algebras over the complex field $C$ (or more generally any field $F$ of characteristic $\neq 2, \neq 3$ ) can be constructed from FreudenthalKantor triple systems. The purpose of this note is to show that the same is also true for Lie superalgebras (for example, $[\mathbf{4}, \mathbf{6}, \mathbf{1 5}]$ ). This fact has been already demonstrated for the case of exceptional Lie superalgebras $D(2,1, \alpha), G(3)$ and $F(4)$ in addition to $\operatorname{osp}(n, 2)$ from some balanced $(-1,-1)$ Freudenthal-Kantor triple systems in a previous paper $([\mathbf{1 0}])$. In this note, we shall construct the rest of the simple Lie superalgebras from some other triple systems for the sake of the completeness. From now on, we shall mainly use the notation and terminology of $([2,10,11])$.

Let $V$ be a finite dimensional superspace over a field $F$ of characteristic not 2 with

$$
L=V_{\overline{0}} \oplus V_{\overline{1}}
$$

in the standard notation. Suppose that $V$ possesses a triple product $V \otimes V \otimes V \rightarrow V$ which is denoted by juxtaposition $x y z$ for $x, y, x \in V$. We assume further that it satisfies a relation

$$
u v(x y z)=(u v x) y z+\varepsilon(-1)^{(u+v) x+u v} x(v u y) z+(-1)^{(u+v)(x+y)} x y(u v z)
$$

for any $u, v, x, y, z \in V$, where $\varepsilon$ is a constant assuming only two values $\varepsilon=+1$ or -1 .

Introducing the left multiplication operator $L: V \otimes V \rightarrow$ End $V$ by

$$
L(x, y) z:=x y z
$$

Received 16th July, 2003

The second author is supported in part by U.S. Department of Energy Contract no. DE-FG0291ER40685.

Copyright Clearance Centre, Inc. Serial-fee code: 0004-9727/04 \$A2.00+0.00. 
equation (1.2) is rewritten as a Lie superalgebra relation of

$$
[L(u, v), L(x, y)]=L(u v x, y)+\varepsilon(-1)^{(u+v) x+u v} L(x, v u y) .
$$

We can readily then construct Lie superalgebras $\operatorname{spl}(n, m)$ and $\operatorname{osp}(n, m)$ as follows. Let (.I.) be a non-degenerate form on $V$ satisfying

$$
\begin{aligned}
\langle x \mid y\rangle & =-\varepsilon(-1)^{x y} y|x\rangle, \\
\left\langle V_{\overline{0}} \mid V_{\overline{1}}\right\rangle & =0 .
\end{aligned}
$$

It is easily seen that the triple products defined by

$$
x y z:=\langle y \mid z\rangle x
$$

and

$$
x y z:=\langle y \mid z\rangle x+\varepsilon(-1)^{x y}\langle x \mid z\rangle y
$$

satisfy equation (1.2), as has been already noted in [10].

The resulting Lie superalgebras for equation (1.6) will take the form of

$$
[L(u, v), L(x, y)]=\langle v \mid x\rangle L(u, y)+\varepsilon(-1)^{(u+v) x+u v}\langle u \mid y\rangle L(x, v)
$$

while equation (1.7) gives

$$
\begin{aligned}
{[L(u, v), L(x, y)]=\langle v| } & x\rangle L(u, y)+\varepsilon(-1)^{u v}\langle u \mid x\rangle L(v, y) \\
& +\varepsilon(-1)^{(u+v) x}\langle u \mid y\rangle L(x, v)+(-1)^{u v+(u+v) x}\langle v \mid y\rangle L(x, u) .
\end{aligned}
$$

If we choose $\varepsilon=-1$, then these reproduce the standard forms of Lie superalgebras $\operatorname{spl}(n, m)$ for equation (1.8) and $\operatorname{osp}(n, m)$ for equation (1.9), where we have set

$$
n=\operatorname{Dim} V_{\overline{0}}, \quad m=\operatorname{Dim} V_{\overline{1}} \text {. }
$$

The other choice of $\varepsilon=+1$ lead to the same except for the fact that we interchange the role of $n$ and $m$.

In the construction given above, $V$ is a superspace with $V_{\overline{1}} \neq 0$ in general. However, we can also construct all Lie superalgebras except possibly for $\operatorname{osp}(n, m)(m>2)$ from some non-super vector spaces (that is, $V_{\overline{1}}=0$ ). We have already shown this fact in [11] for case of $D(2,1 ; \alpha), G(3), F(4)$ and $\operatorname{osp}(n, 2)$.

To this end, let $V$ be hereafter a (non-super) vector space over a field $F$ of characteristic not two. Suppose that a triple product $[., .,$.$] in V$ now satisfies

$$
\begin{gathered}
{[x, y, z]=[y, x, z]} \\
{[x, y, z]+[y, z, x]+[z, x, y]=0} \\
{[u, v,[x, y, z]]=[[u, v, x], y, z]+[x,[u, v, y], z]+[x, y,[u, v, z]]}
\end{gathered}
$$


for any $u, v, x, y, z \in V$. Then $V$ is called an anti-Lie triple system $([3,7,10,11,14])$.

For the triple products, we often use the notation $x y z,[x y z]$ and $\langle x y z\rangle$ according to the traditional custom. Note that if we replace equation (1.11a) by

$$
[x, y, z]=-[y, x, z]
$$

that it defines a more familiar Lie triple system $([5,13])$.

We can now give the canonical standard embedding construction of Lie superalgebras from an anti-Lie triple system as follows. First, we note that the left multiplication operator $L(x, y)$ defined by

$$
L(x, y) z:=[x, y, z]
$$

satisfies a Lie algebra relation of

$$
[L(u, v), L(x, y)]=L([u, v, x], y)+L(x,[u, v, y])
$$

with

$$
L(y, x)=L(x, y)
$$

by equation (1.11c) and (1.11a). We now identify this Lie algebra with the even-part $L_{\overline{0}}$ of a Lie superalgebra

$$
L=L_{\overline{0}} \oplus L_{\overline{1}},
$$

with

$$
\begin{aligned}
& L_{\overline{0}}:=L(V, V), \\
& L_{\overline{1}}:=V .
\end{aligned}
$$

The commutation relations involving $L_{\overline{1}}$ are assumed to satisfy

$$
\begin{aligned}
{[x, y] } & :=L(x, y) \\
{[L(x, y), z] } & :=-[z, L(x, y)]:=[x, y, z]
\end{aligned}
$$

for any $x, y, z \in V$.

Then, it can be easily proved that $L$ is a Lie superalgebra.

One way to construct anti-Lie triple systems is to utilise the balanced $(-1,-1)$ Freudenthal-Kantor triple systems as in [11] for derivations of $D(2,1 ; \alpha), G(3)$ and $F(4)$, which will not be repeated here. Another method which we shall employ in this note below is to consider an anti-Jordan triple system which is a special case of more general $(\varepsilon, \delta)$ Freudenthal-Kantor triple systems $([9,12])$. 
Let $V$ be again a (non-super) vector space. Suppose that it possesses a triple product denoted now by $\langle x y z\rangle$ for $x, y, z \in V$, which satisfies

$$
\begin{aligned}
\langle x y z\rangle & =\delta\langle z y x\rangle \\
\langle u v\langle x y z\rangle\rangle & =\langle\langle u v x\rangle y z\rangle+\varepsilon\langle x\langle v u y\rangle z\rangle+\langle x y\langle u v z\rangle\rangle
\end{aligned}
$$

for constants $\varepsilon, \delta=+1$ or -1 . In particular, the case of $\varepsilon=-1$ and $\delta=1$ gives the familiar Jordan triple systems, while the other case of $\varepsilon=+1$ and $\delta=-1$ is called an anti-Jordan triple system $([\mathbf{7}, \mathbf{1 0}])$.

Introducing the second triple product in any anti-Jordan triple system by

$$
[x, y, z]:=\langle x y z\rangle+\langle y x z\rangle
$$

it can be shown (for example, see $[7,10]$ ) that it defines an anti-Lie triple system. In the following section, we shall construct in this way Lie superalgebras $\operatorname{spl}(n, m), P(n)$ and $Q(n)$.

The remaining case of Cartan-type Lie superalgebras $W(n), S(n) ; \widetilde{S}(n)$ and $H(n)$ will be discussed in section 3 , where we construct them more directly from some appropriate anti-Lie triple system.

In ending this section, we remark that we did not succeed in constructing a general $\operatorname{osp}(n, 2 m)$ Lie superalgebras from a non-super vector space in a simple way except for the case of $\operatorname{osp}(n, 2)$ and $\operatorname{osp}(1,2 m)$. The 1st one for $\operatorname{osp}(n, 2)$ has been already given in terms of $(-1,-1)$ balanced Freudenthat-Kantor triple systems in [11]. For second one $\operatorname{osp}(1,2 m)$, we have the following.

PROPOSITION 1 . Let $V$ be a $2 m$-dimensional vector space with an anti-symmetric nondegenerate bilinear form $\langle x, y\rangle$. Then $(V,[x y z])$ is an anti-Lie triple system with respect to the triple product defined by

$$
[x y z]:=\langle y, z\rangle x+\langle x, z\rangle y .
$$

Thus the canonical standard embedding Lie superalgebra is type osp $(1,2 m)$.

2. Lie superalgebras, $\operatorname{spl}(n, m), P(n)$ and $Q(n)$

Let $V_{n, m}$ be a vector space spaned by all $n \times m$ matrices over a field $F$ of characteristic not 2. For a pair $x \in V_{n, m}$ and $x^{\prime} \in V_{m, n}$, we introduce a large vector space $V$ spanned by all $(n+m) \times(n+m)$ matrices $X$ of the form

$$
X=\left(\begin{array}{cc}
0 & x \\
x & 0
\end{array}\right)
$$

In $V$, we then define a triple product by

$$
\langle X Y Z\rangle:=\left(\begin{array}{cc}
0 & x y^{\prime} z-z y^{\prime} x \\
x^{\prime} y z^{\prime}-z^{\prime} y x^{\prime} & 0
\end{array}\right) \in V
$$


for $X, Y, Z \in V$, where $x y^{\prime} z \in V_{n, m}$ and $x^{\prime} y z^{\prime} \in V_{m, n}$ stand for the standard associative matrix products. Note that we have $x y^{\prime} \in V_{n, n}$ and $x^{\prime} y \in V_{m, m}$ for $x, y \in V_{n, m}$ and $x^{\prime}, y^{\prime} \in V_{m, n}$. It is straightforward to verify that $V$ then becomes an anti-Jordan triple system with respect to this triple product. We can now construct the associated anti-Lie triple system by

$$
\begin{aligned}
{[X, Y, Z] } & :=\langle X Y Z\rangle+\langle Y X Z\rangle \\
& =\left(\begin{array}{cc}
0 & x y^{\prime} z+y x^{\prime} z-z x^{\prime} y-z y^{\prime} x \\
x^{\prime} y z^{\prime}+y^{\prime} x z^{\prime}-z^{\prime} x y^{\prime}-z^{\prime} y x^{\prime} & 0
\end{array}\right),
\end{aligned}
$$

following the prescription described in section 1 . We then calculate the left multiplication operator by

$$
L(X, Y) Z:=[X, Y, Z]=\left[\left(\begin{array}{cc}
x y^{\prime}+y x^{\prime} & 0 \\
0 & x^{\prime} y+y^{\prime} x
\end{array}\right),\left(\begin{array}{cc}
0 & z \\
z^{\prime} & 0
\end{array}\right)\right]
$$

so that we have

$$
L(X, Y)=\operatorname{ad}\left(\begin{array}{cc}
x y^{\prime}+y x^{\prime} & 0 \\
0, & x^{\prime} y+y^{\prime} x
\end{array}\right) .
$$

Here, ad designates the adjoint operation. Since $x, y \in V_{n, m}$ and $x^{\prime}, y^{\prime} \in V_{m, n}$ can be chosen arbitrarily, $x y^{\prime}+y x^{\prime}$ and $x^{\prime} y+y^{\prime} x$ span all $n \times n$ and $m \times m$ matrices, respectively with a constraint

$$
T_{r}^{(n)}\left(x y^{\prime}+y x^{\prime}\right)=T_{r}^{(m)}\left(x^{\prime} y+y^{\prime} x\right),
$$

where $T_{r}^{(n)}$ stands for the trace of $n \times n$ matrix. If $m \neq n$, then $L(V, V)$ can be identified with a diagonal $(n+m) \times(n+m)$ matrix form

$$
L(V, V) \cong\left(\begin{array}{cc}
V_{n, n}, & 0 \\
0, & V_{m, m}
\end{array}\right)
$$

with

$$
\operatorname{str} L(V, V)=0,
$$

where str implies the supertrace. Therefore, the resulting Lie superalgebra $L=L(V, V) \oplus$ $V$ can be also identified as

$$
L \cong\left(\begin{array}{ll}
V_{n, n}, & V_{n, m} \\
V_{m, n}, & V_{m, m}
\end{array}\right)
$$

with the supertrace constraint equation (2.6b). Thus, we have obtained the Lie superalgebra $\operatorname{spl}(n, m)$. 
We have imposed the condition $m \neq n$ in the above for the following reason. For $n=m$, it could happen that for some choices of matrices $x, y, x^{\prime}$, and $y^{\prime}$, we could have

$$
x_{0}=x y^{\prime}+y x^{\prime}, \text { and } x_{0}^{\prime}=x^{\prime} y+y^{\prime} x
$$

to be $n \times n$ identity matrix $E_{n}$ that is,

$$
x_{0}=x_{0}^{\prime}=E_{n}
$$

which is compatible with equation (2.5) for $n=m$. In the case, equation (2.4 b) gives $L\left(X_{0}, Y_{0}\right)=\operatorname{ad}\left(\begin{array}{cc}E_{n} & 0 \\ 0 & E_{n}\end{array}\right)=0$. Therefore, equation (2.7) is now replaced by

$$
L=\left(\begin{array}{ll}
V_{n, n}, & V_{n, n} \\
V_{n, n}, & V_{n, n}
\end{array}\right) / F E_{2 n}
$$

which represents now

$$
\operatorname{spl}(n, n) / F E_{2 n}(\text { see }[15]) \text {. }
$$

We next proceed for constructions of strange Lie superalgebras $P(n)$ and $Q(n)$. Hereafter we restrict ourselves to the case of $m=n$. We first consider a choice of $x^{\prime}=x$ in equation (2.1) with $\operatorname{tr} x=0$ so that

$$
V=\left\{X \mid X=\left(\begin{array}{ll}
0 & x \\
x & 0
\end{array}\right), \operatorname{tr} x=0, x \in V_{n, n}\right\}
$$

Equation (2.2) becomes then

$$
\langle X Y Z\rangle=\left(\begin{array}{cc}
0 & x y z-z y x \\
x y z-z y x & 0
\end{array}\right) \in V
$$

since we have $\operatorname{tr}(x y z-z y x)=0$. Moreover, equation $(2.4 \mathrm{~b})$ is rewritten as

$$
L(X, Y)=\operatorname{ad}\left(\begin{array}{cc}
x y+y x & 0 \\
0 & x y+y x
\end{array}\right)
$$

so that the resulting Lie superalgebra $L=L(V, V) \oplus V$ can be identified in a similar way with

$$
L=\left\{\left(\begin{array}{ll}
u & x \\
x & u
\end{array}\right) \mid \operatorname{tr} x=0, u, x \in V_{n, n}\right\} / F E_{2 n}
$$

which realises the Lie superalgebra $Q(n-1)$ (or $d(n)$ in the notation of [15]). 
We finally consider the choice of ${ }^{t} x=x$ and ${ }^{t} x^{\prime}=-x^{\prime}$ where ${ }^{t} x$ stands for the transpose matrix of $x$. We put

$$
V=\left\{X \mid X=\left(\begin{array}{cc}
0 & x \\
x^{\prime} & 0
\end{array}\right),{ }^{t} x=x,{ }^{t} x^{\prime}=-x^{\prime}, x, x^{\prime} \in V_{n, n}\right\}
$$

with

$$
\langle X Y Z\rangle=\left(\begin{array}{cc}
0 & x y^{\prime} z-\dot{z} y^{\prime} x \\
x^{\prime} y z^{\prime}-z^{\prime} y x^{\prime} & 0
\end{array}\right) \in V
$$

since

$$
\begin{aligned}
{ }^{t}\left(x y^{\prime} z-z y^{\prime} x\right) & =x y^{\prime} z-z y^{\prime} x, \\
{ }^{t}\left(x^{\prime} y z^{\prime}-z^{\prime} y x^{\prime}\right) & =-\left(x^{\prime} y z^{\prime}-z^{\prime} y x^{\prime}\right) .
\end{aligned}
$$

Moreover, we note $x^{\prime} y+y^{\prime} x=-{ }^{t}\left(x y^{\prime}+y x^{\prime}\right)$ and hence $L(X, Y)$ is rewritten as

$$
L(X, Y)=\operatorname{ad}\left(\begin{array}{cc}
u & 0 \\
0 & -{ }^{t} u
\end{array}\right)
$$

for $u=x y^{\prime}+y x^{\prime} \in V_{n, n}$. Then the resulting Lie superalgebra is $P(n-1)$ (or $\left.b(n)\right)$ when we note

$$
L=\left\{\left(\begin{array}{cc}
u & x \\
x^{\prime} & -{ }^{t} u
\end{array}\right) \mid{ }^{t} x=x, \quad{ }^{t} x^{\prime}=-x^{\prime}, x, x^{\prime}, u \in V_{n, n}\right\} .
$$

Summarising the results of above and Section 1, we have the following.

Propos ITION 2. Let $(V,\langle x y z\rangle)$ be an anti-Jordan triple system. Then $(V,[x y z])$ is an anti-Lie triple system with respect to the new product defined by

$$
[x y z]:=\langle x y z\rangle+\langle y x z\rangle
$$

In particular, we can obtain the simple Lie superalgebras $\operatorname{spl}(n, m), P(n)$ and $Q(n)$ from anti-Jordan triple systems by means of the canonical standard embedding Lie superalgebra associated with $V$.

\section{Cartan-type Lie Superalgebras}

All Cartan-type Lie superalgebras can be constructed directly from some anti-Lie triple systems. Before going into its details, we first note the following.

Suppose that a Lie superalgebra

$$
L=L_{\overline{0}} \oplus L_{\overline{1}}
$$


satisfies conditions

$$
\left[L_{\overline{\mathbf{1}}}, L_{\overline{\mathbf{1}}}\right]=L_{\overline{0}}
$$

$$
\text { ad } L_{\overline{0}} \text { is isomorphic to } L_{\overline{0}} \text { as Lie algebras. }
$$

Setting $V=L_{\overline{1}}$, we introduce, next, a triple product in $V$ by

$$
[x, y, z]:=[[x, y], z]
$$

for $x, y, z \in V$. This defines an anti-Lie triple system. Moreover its canonial construction of a Lie superalgebra as described in section 1 gives a Lie superalgebra isomorphic to the original $L$. All simple Cartan-type Lie superalgebras $W(n), S(n), \widetilde{S}(n)$, and $H(n)$ satisfy the desired conditions so that they can be surely constructed in terms of the corresponding anti-Lie triple systems.

Let $\Gamma(n)$ be the Grassmann algebra generated by $n$ anti-commuting variables $\theta_{1}, \theta_{2}, \cdots, \theta_{n}$. It is well-known then that

$$
W(n):=\left\{X=\sum_{i=1}^{n} \tilde{X}_{i} \frac{\partial}{\partial \theta_{i}} \mid \widetilde{X}_{i} \in \Gamma(n)\right\}
$$

is the Lie superalgebra consisting of all superderivations of $\Gamma(n)$.

Therefore setting

$$
V=W_{\overline{1}}(n)=\left\{X=\sum_{i=1}^{n} \tilde{X}_{i} \frac{\partial}{\partial \theta_{i}} \mid \tilde{X}_{i} \in \Gamma(n), \text { grade } \tilde{X}_{i}=\text { even }\right\},
$$

and introducing the triple product

$$
\begin{aligned}
{[X, Y, Z] } & :=(X Y+Y X) Z-Z(X Y+Y X) \\
& =[[X, Y], Z]
\end{aligned}
$$

in $V$, this gives an anti-Lie triple system whose canonical construction leads to $W(n)$.

We may also note that

$$
\begin{aligned}
{[X, Y, Z] } & =\sum_{i=1}^{n} \widetilde{W}_{i} \frac{\partial}{\partial \theta_{i}} \\
\widetilde{W}_{i} & =\sum_{j, k=1}^{n}\left\{\left(X_{j} \frac{\partial}{\partial \theta_{j}} Y_{k}+Y_{j} \frac{\partial}{\partial \theta_{j}} X_{k}\right) \frac{\partial}{\partial \theta_{k}} Z_{i}-Z_{k} \frac{\partial}{\partial \theta_{k}}\left(X_{j} \frac{\partial}{\partial \theta_{j}} Y_{i}+Y_{j} \frac{\partial}{\partial \theta_{j}} X_{i}\right)\right\}
\end{aligned}
$$

for $X, Y, Z \in V\left(=W_{\overline{\mathrm{I}}}(n)\right)$.

We next consider a Lie supersubalgebra of $W(n)$ given by

$$
S(n)=\left\{X \mid X=\sum_{j=1}^{n} \tilde{X}_{i} \frac{\partial}{\partial \theta_{i}}, \sum_{i=1}^{n} \frac{\partial}{\partial \theta_{i}} \tilde{X}_{i}=0\right\} .
$$


We repeat the same procedure to find a Lie superalgebra isomorphic to $S(n)$ by considering an anti-Lie triple system constructed from its odd part $S_{\overline{1}}(n)$.

Similarly, we set

$$
\widetilde{S}(n)=\left\{X \mid X=\sum_{i=1}^{n} \widetilde{X}_{i} \frac{\partial}{\partial \theta_{i}}, \sum_{i=1}^{n} \frac{\partial}{\partial \theta_{i}}\left(1+\theta_{1} \theta_{2} \cdots \theta_{n}\right) \tilde{X}_{i}=0\right\},
$$

where $n$ is now assumed to be an even integer.

We note that $\widetilde{S}(n)$ is characterised also as a linear combination of elements of the forms

$$
X=\left(1-\theta_{1} \theta_{2} \cdots \theta_{n}\right) \sum_{i=1}^{n} a_{i} \frac{\partial}{\partial \theta_{i}}, a_{i} \in F
$$

and

$$
X=\sum_{i, j=1}^{n}\left(\frac{\partial}{\partial \theta_{i}} \widetilde{X}_{i j}\right) \frac{\partial}{\partial \theta_{i}}
$$

where $\widetilde{X}_{i j} \in \Gamma(n)$ satisfies

$$
\widetilde{X}_{i j}=\widetilde{X}_{j i}, \text { grade } \widetilde{X}_{i j} \geqslant 2 .
$$

We must verify the validity of conditions equation (3.2). For example, for the case of

$$
X_{0}=\left(1-\theta_{1} \theta_{2} \cdots \theta_{n}\right) \sum_{i=1}^{n} a_{i} \frac{\partial}{\partial \theta_{i}} \in V=\widetilde{S}_{\overline{1}}(n)
$$

and

$$
Y_{0}=\left(1-\theta_{1} \theta_{2} \cdots \theta_{n}\right) \sum_{i=1}^{n} b_{i} \frac{\partial}{\partial \theta_{i}} \in V=\widetilde{S}_{\overline{1}}(n)
$$

for some $a_{i}, b_{i} \in F$, we calculate

$$
\begin{aligned}
{\left[X_{0}, Y_{0}\right] } & =\sum_{i, j=1}^{n}\left(\frac{\partial}{\partial \theta_{j}} \widetilde{Z}_{i j}\right) \frac{\partial}{\partial \theta_{i}} \in \widetilde{S}_{\overline{0}}(n) \\
\tilde{Z}_{i j} & =-\left(a_{i} b_{j}+a_{j} b_{i}\right) \theta_{1} \theta_{2} \cdots \theta_{n} .
\end{aligned}
$$

From these, we can verify the validity of the conditions equation (3.2).

Finally, let us consider a Lie superalgebra $\widetilde{H}(n)$ and we put

$$
\tilde{H}(n)=\left\{X=\sum_{i=1}^{n} \tilde{X}_{i} \frac{\partial}{\partial \theta_{i}} \mid \tilde{X}_{i}=\frac{\partial}{\partial \theta_{i}} X_{0}, X_{0} \in \Gamma(n)\right\} .
$$

However, $\widetilde{H}(n)$ is not simple and we restrict to its Lie supersubalgebra $H(n)$ given by

$$
H(n):=\left\{X \mid X \in \tilde{H}(n), \text { grade } X_{0} \leqslant n-1\right\} .
$$


We conclude that

$$
H(n)=[\tilde{H}(n), \tilde{H}(n)]
$$

since for $X, Y \in \widetilde{H}(n)$ with

$$
X=\sum_{i=1}^{n} \frac{\partial X_{0}}{\partial \theta_{i}} \frac{\partial}{\partial \theta_{i}}, Y=\sum_{i=1}^{n} \frac{\partial Y_{0}}{\partial \theta_{i}} \frac{\partial}{\partial \theta_{i}}
$$

we calculate

$$
Z=[X, Y]=\sum_{i=1}^{n} \frac{\partial Z_{0}}{\partial \theta_{i}} \frac{\partial}{\partial \theta_{i}}
$$

with

$$
Z_{0}=(-1)^{X+1} \sum_{j=1}^{n} \frac{\partial}{\partial \theta_{i}}\left(X_{0} \frac{\partial Y_{0}}{\partial \theta_{j}}\right)
$$

whose grade is obviously less than $n$.

Summarising the results of above and Section 1, we have the following.

Proposition 3. Let $V$ be an anti-Lie triple system and $L(V, V)$ be the Lie algebras of inner derivations of $V$. Then $L(V):=L(V, V) \oplus V$ is a Lie superalgebra with respect to

$$
\left[D+X, D^{\prime}+Y\right]:=\left[D, D^{\prime}\right]+D Y-D^{\prime} X+L(X, Y)
$$

where $D, D^{\prime} \in L(V, V), X, Y \in V$

In particular, we can obtain the simple Lie superalgebras $W(n), S(n), \widetilde{S}(n), H(n)$ by means of the canonical standard embedding.

In conclusion, there exists a way to construct all simple Lie superalgebras from some triple systems (for example, anti-Lie triple systems) without using the terms of supertriple systems except possibly $\operatorname{osp}(n, m)$. That is, by means of non-super vector spaces.

\section{REFERENCES}

[1] H. Asano, 'Symplectic triple systems and simple Lie algebras Kokyuroku', RIMS, Kyoto University 308 (1977), 41-54.

[2] A. Elduque, N. Kamiya and S. Okubo, 'Simple $(-1,-1)$ balanced Freudenthal-Kantor triple systems', Glasgow Math. J. 45 (2003), 353-372.

[3] J. Faulkner and J.C. Ferrar, 'Simple anti-Jordan pairs', Comm. Alg. 8 (1980), 993-1013.

[4] L. Frappat, A. Sciarrino and P. Sorba, Dictionary on Lie superalgebras (Academic Press, San Diego, CA, 2000).

[5] N. Jacobson, 'Structure and representations of Jordan qlgebras', Amer. Math. Soc. Colloq. Publications 39 (American Mathematical Society, Providence, RI).

[6] V.G. Kac, 'Classification of Z-graded Lie superalgebras and simple Jordan superalgebras', Comm. Algebra 5 (1977), 1375-1400. 
[7] N. Kamiya, 'A construction of Anti-Lie triple systems from a class of triple systems', Mem. Fac. Sci. Shimane Univ. 22 (1988), 51-62.

[8] N. Kamiya, 'The construction of all simple Lie algebras over $C$ from balanced Freudenthal-Kantor triple system', in Contribute to general algebras 7 (Holder-Pichler-Tempsky, Wien, 1991), pp. 205-213.

[9] N. Kamiya, 'On Freudenthal-Kantor triple systems and generalised structure algebras', in Nonassociative algebra and its applicaton, Math. Appl. 303 (Kluwer Acad. Publ., Dordrecht, 1994), pp. 198-203.

[10] N. Kamiya and S. Okubo, 'On $\delta$-Lie Supertriple Systems Associated with $(\varepsilon, \delta)$ Freudenthal-Kantor Supertriple Systems', Proc. Edinburugh Math. Soc. 43 (2000), 243-260.

[11] N. Kamiya and S. Okubo, 'Construction of Lie superalgebras $D(2,1 ; \alpha), G(3)$ and $F(4)$ from some triple systems', Proc. Edinburugh Math.Soc. 46 (2003), 87-98.

[12] N. Kamiya and S. Okubo, 'On generalised Freudenthal-Kantor triple systems and Yang-Baxter equations', Proc. XXIV International Coll. Group Theoretical Methods in Physics (2002) (to appear).

[13] W.G. Lister, 'A Structure Theory of Lie Triple System', Trans. Amer. Math. Soc. 72 (1952), 217-242.

[14] S. Okubo and N. Kamiya, 'Quasi-classical Lie superalgebras and Lie supertriple systems', Comm. Algebra 30 (2002), 3825-3850.

[15] M. Scheunert, The theory of Lie superalgebras, Lecture Notes in Maths 716 (Springer-Verlag, Berlin, 1970).

Department of Mathematics

The University of Aizu

Aizu-Wakamatsu 965-8580

Fukushima

Japan

e-mail: kamiya@u-aizu.ac.jp
Department of Physics and Astronomy

The University of Rochester

Rochester, N.Y. 14627

United States of America

e-mail: okubo@pas.rochester.edu 